\title{
Caracterização do atendimento após implantação do acolhimento, avaliação e classificação de risco em hospital público
}

\begin{abstract}
Characterization of the care assistance after the implementation of the embracement, evaluation and classification of risk in a public hospital
\end{abstract}

Caracterización de la atención en salud después de la implantación de la acogida com evaluación y la clasificación de riesgo en un hospital público

Mariana Angela Rossaneis ${ }^{\mathrm{I}}$, Maria do Carmo Lourenço Haddad ${ }^{\mathrm{II}}$, Fabiane Gorni Borsato ${ }^{\mathrm{III}}$, Marli Oliveira Vannuchi ${ }^{\mathrm{IV}}$, Andreza Daher Delfino Sentone ${ }^{\mathrm{V}}$

\section{RESUMO}

O estudo teve por objetivo descrever a implantação do acolhimento com avaliação e classificação de risco (AACR) em um hospital público de média complexidade e caracterizar o atendimento após a implantação dessa estratégia. Trata-se de um estudo descritivo exploratório realizado em duas etapas: na primeira foram descritas as estratégias para implantação do AACR e na segunda foi levantada a quantidade de consultas médicas realizadas durante quatro meses após a implantação e comparadas com os meses que a precederam. A estruturação do grupo multiprofissional e a construção do planejamento estratégico situacional proporcionaram a operacionalização do processo. Após a implantação do AACR, 36\% dos usuários foram avaliados pelo enfermeiro e encaminhados a outros serviços o que resultou em redução de $69 \%$ nos atendimentos médicos. O AACR agilizou o atendimento de pacientes que necessitaram de tratamento imediato, considerando o risco à saúde e o grau de sofrimento.

Descritores: Acolhimento; Humanização da Assistência; Serviço Hospitalar de Emergência; Serviço Hospitalar de Enfermagem.

\section{ABSTRACT}

The study aimed to describe the implementation of embracement with an evaluation and classification of risk (EECR) in a public hospital of medium complexity and characterize the attendance after the implementation of this strategy. This is an exploratory descriptive study developed into two stages: at the first, the EECR implementation strategies were described, at the second, the amount of medical consultations were numbered during the period of four months after implantation and compared with the months that preceded them. The multi professional group structuring and the construction of the situational strategic planning provided the process operationalization. After implementation of EECR, 36\% of users were assessed by the nurse and referred to other services, which resulted in $69 \%$ of reduction in medical care. The EECR streamlined the care of patients who required immediate treatment, considering the health risk and the suffering degree.

Descriptors: User Embracement; Humanization of Assistance; Emergency Service, Hospital; Nursing Service, Hospital.

\section{RESUMEN}

El estudio objetivó describir la implementación de la acogida con evaluación y clasificación de riesgo (AECR) en un hospital público de mediana complejidad y caracterizar la atención al público después de la aplicación de esta estrategia. Se trata de un estudio descriptivo y exploratorio, realizado en dos etapas: en la primera fueron descritas las estrategias para la implementación de la AECR y en la segunda se levantó la cantidad de consultas médicas realizadas durante cuatro meses después de la implantación y comparándolas con los meses que la precedieron. La estructuración del grupo multiprofesional y la construcción del planeamiento estratégico situacional proporcionarán la ejecución del proceso. Después de la implantación de la AECR, el 36\% de los usuarios fueron evaluados por un enfermero y destinados a otros servicios, lo que resultó en una disminución del $69 \%$ en los atendimientos médicos. La AECR proporcionó mayor agilidad en los atendimientos de pacientes que necesitaban tratamiento inmediato, considerando el riesgo a la salud y el grado de sufrimiento.

Descriptores: Acogimiento; Humanización de la Atención; Servicio de Urgencia en Hospital; Servicio de Enfermería en Hospital.

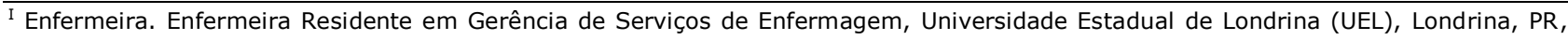
Brasil. E-mail: rossaneis@hotmail.com.

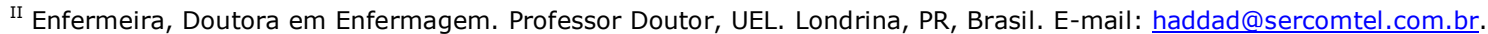

III Enfermeira. Enfermeira Residente em Gerência de Serviços de Enfermagem. Londrina, PR, Brasil. E-mail: fabigorni@hotmail.com.

IV Enfermeira, Doutora em Enfermagem. Professor Doutor, UEL. Londrina, PR, Brasil. E-mail: vannuchi@sercomtel.com.br.

${ }^{\vee}$ Enfermeira, Mestre em Enfermagem. Londrina, PR, Brasil. E-mail: andrezadaher@sercomtel.com.br.
} 


\section{INTRODUÇÃo}

Instituída pelo Ministério da Saúde (MS) em 2003, a Política Nacional de Humanização da Atenção e Gestão do Sistema Único de Saúde (SUS), o HumanizaSUS, tem o objetivo de tornar a humanização um movimento capaz de fortalecer o SUS como política pública de saúde, garantindo que a assistência seja baseada em suas diretrizes - igualdade, universalidade e equidade ${ }^{(1)}$.

Os valores que norteiam essa política são a autonomia e o protagonismo dos sujeitos, a coresponsabilidade entre eles, o estabelecimento de vínculos solidários, a construção de redes de cooperação e a participação coletiva no processo de gestão( ${ }^{(2)}$.

Uma das diretrizes de maior relevância nesta política é o acolhimento, definido como processo de práticas de saúde que implicam na responsabilização da equipe de saúde pelo usuário. Para que essa diretriz seja aplicada é necessária a qualificação do profissional para lidar com as singularidades dos sujeitos, saber ouvir a queixa do usuário e pactuar a estratégia mais adequada a cada individuo, garantindo assistência integral, humanizada, com resolutividade no atendimento e acesso a serviços externos, visando a transversalização da rede de atenção à saúde ${ }^{(1)}$.

Entretanto, não é este o tipo de acolhimento que encontramos na maioria dos serviços de saúde de emergência. Tradicionalmente, os prontos-socorros funcionam como porta de entrada da rede de saúde, onde são atendidos casos clínicos graves, com risco de morte, e usuários com quadros clínicos leves ou moderados que não conseguem assistência na rede de cuidados primários ${ }^{(3)}$.

Além disso, a dificuldade de acesso à atenção ambulatorial especializada e à assistência hospitalar também impacta no atendimento dos serviços de emergência, que passa a ser a principal via de acesso a especialidades e tecnologias médicas, tornando-se um depósito de problemas não resolvidos ${ }^{(4)}$. Por estas razões, os hospitais de emergência sofrem, mais que outras unidades, os efeitos da falta de investimento no sistema público de saúde e de sua crise de governabilidade ${ }^{(5)}$.

No Brasil, a maioria dos hospitais públicos funciona acima de sua capacidade máxima, com taxa de ocupação de leitos superior a $100 \%$, número reduzido de recursos humanos, profissionais sem capacitação, excesso de demanda e demanda inadequada, déficit financeiro, gerenciamento precário de recursos, número de leitos insuficientes e sem planejamento efetivo ${ }^{(4)}$.
Essa crise torna-se ainda mais grave pela falta de um sistema de classificação de risco, uma vez que o atendimento é realizado por ordem de chegada, sem avaliação do quadro clínico do usuário, causando sérios prejuízos a sua saúde pela intervenção tardia. Nesse tipo de serviço o acolhimento é sinônimo de ambiente de recepção administrativa ou funciona apenas como triagem que organiza filas por ordem de chegada sem avaliar o grau de sofrimento do usuário e/ou potencial de risco à saúde ${ }^{(6)}$

Para resolver esta situação o HumanizaSuS prevê a implantação do acolhimento com avaliação e classificação de risco (AACR) nos serviços de saúde de emergência. O objetivo é acolher e atender a demanda de acordo com a avaliação de risco e garantir acesso referenciado aos demais níveis de assistência ${ }^{(1)}$.

A classificação de risco é uma ferramenta necessária para organizar o fluxo de atendimento nos serviços de saúde de emergência e proporciona assistência mais resolutiva e humanizada àqueles em situações de risco a saúde. Dessa forma, entende-se a descrição das estratégias utilizadas para implantação do AACR em um hospital público e a análise do impacto no número de consultas médicas possam contribuir com outras instituições de saúde na reorganização do fluxo de atendimento dos usuários nos serviços de emergência.

Este estudo teve por objetivo descrever a implantação do acolhimento com avaliação e classificação de risco em um hospital público de média complexidade e caracterizar o atendimento após a implantação dessa estratégia.

\section{MÉTODOS}

Trata-se de um estudo descritivo exploratório, cujas informações foram levantadas nos registros do prontosocorro de um hospital público de média complexidade, entre os meses de agosto de 2009 a março de 2010 .

A instituição possui 65 leitos, 30 para internação em clínica médica adulto, 14 clínica pediátrica, 15 leitos para pacientes adultos cirúrgicos e seis para pacientes infantis cirúrgicos.

O pronto-socorro funciona 24 horas e atente pacientes encaminhados pelo Serviço de Atendimento Móvel de Urgência (SAMU), Serviço Integrado de Atendimento ao Trauma em Emergência (SIATE), Unidades Básicas de Saúde (UBS) e demanda espontânea. Os atendimentos são realizados no ProntoSocorro Médico (PSM), Pronto-Socorro Cirúrgico (PSC), Pronto-Socorro Ortopédico (PSORT) e Pronto-Socorro 
Pediátrico (PSP). Neste trabalho foram excluídos os dados do PSP, pois nessa instituição não é realizado o encaminhamento de menores de 12 anos de idade a outros serviços.

O centro cirúrgico realiza cirurgias eletivas de pequeno e médio porte.

O estudo foi realizado em duas etapas. Na primeira etapa foram descritas as estratégias utilizadas para implantação do AACR pelas enfermeiras, residentes de enfermagem e docente, autoras deste estudo, que participaram do Grupo de Trabalho que organizaram a implantação.

$\mathrm{Na}$ segunda etapa foi levantada a quantidade de consultas médicas realizadas nos PSM, PSC e PSORT durante quatro meses após a implantação do AACR, entre os meses de dezembro de 2009 a março de 2010 e comparou-se com o número de atendimentos médicos dos quatro meses que precederam a avaliação de risco, ou seja, entre os meses de agosto a novembro de 2009.

O número de consultas mensais por especialidade e o número de encaminhamentos mensais realizados após a avaliação e classificação de risco pelo enfermeiro foram coletados por meio de um instrumento elaborado no programa Microsoft Excel 2007.

A análise dos dados obtidos nos quatro primeiros meses após a implantação em comparação com os quatro meses que a precederam foram apresentados em gráficos com análise crítica e descritiva.

Para a realização do estudo obteve-se a autorização da Direção Clínica, da Direção Administrativa e Coordenação de Enfermagem da instituição, sendo dispensada pela mesma a submissão do projeto ao Comitê de Ética.

\section{RESULTADOS E DISCUSSÃO}

A implantação do AACR surgiu da necessidade de reorganizar o fluxo de atendimento no pronto-socorro da instituição do estudo, de forma que os casos de maior gravidade fossem identificados e priorizados, promovendo a humanização e resolutividade na assistência.

Com este intuito, em julho de 2009 o hospital implantou um Programa de Melhoria da Qualidade do Atendimento e Humanização no Pronto-Socorro, de acordo com os princípios do Humanizasus.

A primeira ação deste programa foi a implantação do AACR no pronto-socorro com o apoio dos profissionais e gestores da instituição. Abrir o front da melhoria da qualidade dos serviços é um compromisso de profissionais e gestores que atuam diretamente nos serviços de saúde ${ }^{(7)}$.

Para a implantação da AACR formou-se um Grupo de Trabalho de Humanização, constituído por equipe multiprofissional, composta por enfermeiros, médicos, assistente social e profissionais do setor administrativo.

A formação deste grupo teve o objetivo de direcionar as atividades para implantação do acolhimento. Para isto, foi estabelecida uma gestão participativa entre os membros e todas as decisões foram tomadas em equipe, visando atender as necessidades do serviço de uma maneira geral. Este estilo de gerenciamento participativo prioriza a tomada de decisões por consenso, procura contemplar todas as opiniões e pontos de vista dos integrantes de uma equipe de trabalho ${ }^{(8)}$.

O grupo de trabalho estabeleceu diversas estratégias de comunicação com o objetivo de otimizar o processo de implantação. Foram realizadas reuniões semanais entre os integrantes do grupo, reuniões mensais com as Diretorias (Geral, Clínica e Administrativa) e reuniões com representantes de cada categoria profissional e da comunidade. A comunicação pode ser visualizada como ferramenta de mudança e melhoria do cuidado ${ }^{(8)}$.

Optou-se pela elaboração de um planejamento estratégico situacional (PES) para identificar os problemas e traçar planos e metas para capacitar os profissionais e adequar o pronto-socorro para a implantação do AACR. O PES é um método que estimula o diálogo e a reflexão sobre os problemas que incidem em uma realidade, visando prever situações e alternativas, antecipar possibilidades de decisão e preparar estratégias para a obtenção de governabilidade sobre as mesmas $^{(9)}$.

O PES elaborado pelo grupo de trabalho identificou os seguintes problemas: desorganização no fluxo de atendimento no pronto-socorro; inadequação da estrutura física; falta de capacitação dos profissionais de saúde em atendimentos de urgência e emergência; consultas médicas realizadas por ordem de chegada sem levar em consideração o grau de risco; demanda espontânea superior a capacidade de atendimento.

Após a identificação dos problemas foi estabelecido um plano de trabalho com as seguintes ações: adequação da estrutura física e sinalização no prontosocorro para as áreas correspondentes aos eixos vermelho, amarelo, verde e azul; elaboração do protocolo de AACR em conjunto com os profissionais de saúde que atuam no pronto-socorro; reuniões com os coordenadores dos serviços da rede de atenção à saúde 
do município para divulgação do novo fluxograma de atendimento; capacitação dos trabalhadores sobre o AACR e assistência em urgência e emergência; conscientização dos usuários.

A elaboração do protocolo de AACR foi baseada no protocolo descrito pelo MS com alguns ajustes para adequá-lo a realidade do hospital onde o estudo foi realizado. Este protocolo foi construído por meio de consensos estabelecidos entre a equipe médica $e$ equipe de enfermagem para avaliar e classificar a gravidade ou o potencial de agravamento de cada caso, assim como o grau de sofrimento do usuário.

O projeto de implantação do AACR foi apresentado e aprovado no Conselho Municipal de Saúde e, posteriormente, divulgado aos coordenadores das Unidade Básicas de Saúde (UBSs) e aos outros serviços de saúde na região.

A capacitação abrangeu todos os trabalhadores da instituição, ou seja, profissionais de enfermagem, médicos, funcionários responsáveis pela portaria, recepção, e telefonia, técnicos administrativos e auxiliares operacionais. Visto que o acolhimento deve ser entendido e aderido por todos os profissionais envolvidos no processo de produção em saúde por se tratar de uma postura ética que não pressupõe hora ou profissional específico para fazê-lo, é o que o diferencia de triagem, pois se constitui numa ação de inclusão que não se esgota na etapa da recepção, mas que deve ocorrer em todos os locais e momentos do serviço de saúde ${ }^{(2)}$.

A capacitação foi realizada em dois dias, com carga horária de 16 horas. Foram utilizadas metodologias pedagógicas ativas e problematizadoras. Problematizar e conscientizar a humanização do ambiente, mais especificamente a partir do trabalhador, implica uma reflexão crítica e dialógica acerca dos princípios e valores que norteiam a prática dos profissionais, de modo a assumirem sua condição de sujeitos e agentes de transformação(10)

O evento foi realizado após a jornada de trabalho e a carga horária da capacitação foi concedida como horas extras aos trabalhadores. Os temas discutidos foram: a Política Nacional de Humanização da Atenção e Gestão do Sistema Único de Saúde; o processo de trabalho nos serviços de urgência e emergência associado ao princípio da ambiência; apresentação do protocolo de acolhimento com avaliação e classificação de risco; estudos de caso baseados na realidade da demanda do hospital; cronograma de implantação do acolhimento com avaliação e classificação de risco no hospital onde o estudo foi realizado.

Também participaram da capacitação representantes de UBSs e outras instituições hospitalares do município, com o objetivo de divulgar a Política Nacional de Humanização da Atenção e Gestão do Sistema Único de Saúde e o novo fluxograma de atendimento do hospital.

Essa parceria proporcionou o elaboração de ações para um sistema de referência e contra-referência entre os serviços. A referência e contra-referência favorecem a prestação da atenção integral ao usuário, garantido que o paciente seja acompanhado pela rede de atenção primária após a alta hospitalar ou retorne ao hospital caso haja necessidade. A interação entre serviços auxilia na redução de casos de reinternação hospitalar e previne o agravamento da patologia do usuário por falta de assistência.

O sucesso em reduzir a superlotação nos serviços de saúde de emergência só é possível se for reorganizado o fluxo de atendimento em toda a rede de saúde, como a participação de todos os serviços da rede, pois o grande número de atendimentos nos serviços de emergência indica baixo desempenho do sistema de saúde como um todo $^{(11)}$

O grupo de trabalho atuou junto à comunidade externa por meio de palestras e discussões em grupo sobre o AACR. A divulgação do processo foi realizada com banners e folders explicativos sobre o AACR e houve a colaboração da imprensa local. Humanizar a assistência em saúde implica dar lugar tanto à palavra do usuário quanto à palavra dos profissionais da saúde, de forma que possam fazer parte de uma rede de diálogo, que pense e promova as ações, campanhas, programas e políticas assistenciais ${ }^{(12)}$.

A implantação do AACR no hospital estava prevista para o mês de agosto de 2009. Mas, devido à determinação da Secretaria Municipal de Saúde o hospital tornou-se uma das instituições de referência no município para o atendimento de pacientes com suspeita da gripe A H1N1 e, portanto, não poderia encaminhar pacientes para atendimento nas UBSs, atrasando a implantação do processo. Somente em dezembro de 2009, o grupo de trabalho retomou as atividades e houve a implantação do AACR no hospital.

Todos os profissionais que atuam na instituição fazem parte do acolhimento dos usuários. Já a avaliação e classificação de risco são realizadas pelo enfermeiro, com respaldo médico, segundo o protocolo estabelecido, e registradas na ficha de atendimento. A utilização de 
protocolos para embasar a classificação de risco oferece respaldo legal para atuação segura do enfermeiro(13).

Após a avaliação do enfermeiro o usuário é classificação nas seguintes cores, segundo o grau de risco: vermelho, pacientes com risco de morte, atendimento imediato; amarelo, pacientes críticos e semi-críticos, atendimento imediato; verdes, casos não urgentes, atendimento o mais rápido possível; azuis, consultas de baixa complexidade, atendimento de acordo com o horário de chegada.

Os pacientes permanecem na sala de espera após a avaliação e são reavaliados enquanto aguardam o atendimento.

Por tratar-se de um hospital de média complexidade, os pacientes classificados com as cores vermelho e amarelo recebem atendimento imediato e após a estabilização do quadro clínico são encaminhados para serviços que prestam assistência alta complexidade. Mas, devido à superlotação dos hospitais de alta complexidade, alguns pacientes permanecem no hospital por mais tempo que o previsto.
Já os pacientes classificados com a cor azul são encaminhados para UBS com um documento de referência. Caso o paciente recuse o encaminhamento, recebe atendimento médico de acordo com a ordem de chegada.

$\mathrm{Na}$ segunda etapa do estudo analisou-se o impacto das consultas médicas na instituição após a implantação do AACR.

Percebeu-se que entre os meses de agosto a novembro de 2009, período anterior a implantação AACR, 21.065 usuários obtiveram atendimento médico, no PSM, PSC e PSORT. Em média, 5.266 consultas médicas/mês e 174 consultas médicas/dia. Já entre os meses de dezembro de 2009 a março de 2010, período avaliado após a implantação do sistema de classificação de risco, o número de atendimentos médicos foi reduzido a 7.753 consultas, em média 1.938 consultas médicas/mês e 64 consultas médicas/dia (Gráfico 1). Ou seja, houve queda de $64 \%$ no número de consultas médicas após a implantação do AACR.

Gráfico 1: Consultas médicas realizadas no pronto-socorro de um hospital de média complexidade, entre os meses de agosto de 2009 a março de 2010. Londrina, PR.

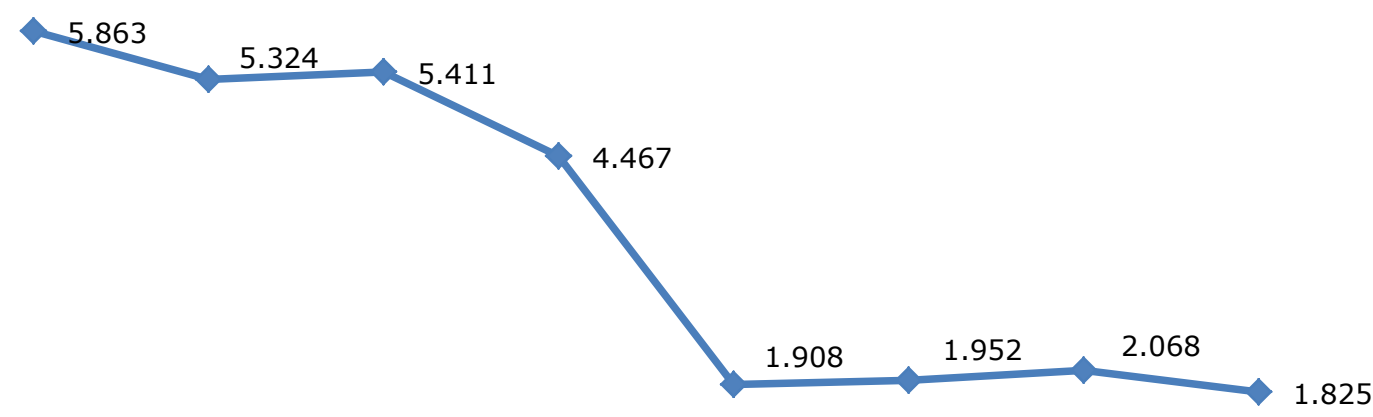

\begin{tabular}{|l|l|l|l|l|l|}
\hline ago/09 & set/09 & out/09 nov/09 & dez/09 & jan/10 & fev/10
\end{tabular}

Fonte: Núcleo de Informação do Hospital, Londrina, PR, 2010.

No PSM a queda no número de consultas médicas foi mais impactante. Neste setor foram atendidos 18.007 usuários, no período avaliado anterior a avaliação de risco, com média de 4.501 consultas médicas/mês e 148 consultas médicas/dia. Após a implantação do acolhimento, em quatro meses o número dos atendimentos médicos no PSM foi 5.602 consultas, com média de 1.400 consultas médicas/mês e 46 consultas médicas/dia (Gráfico 2). Observou-se redução de $69 \%$ no número de atendimentos ao compararmos os dois períodos. 
Gráfico 2: Consultas médicas realizadas no pronto-socorro médico de um hospital de média complexidade, entre os meses de agosto de 2009 a março de 2010. Londrina, PR.

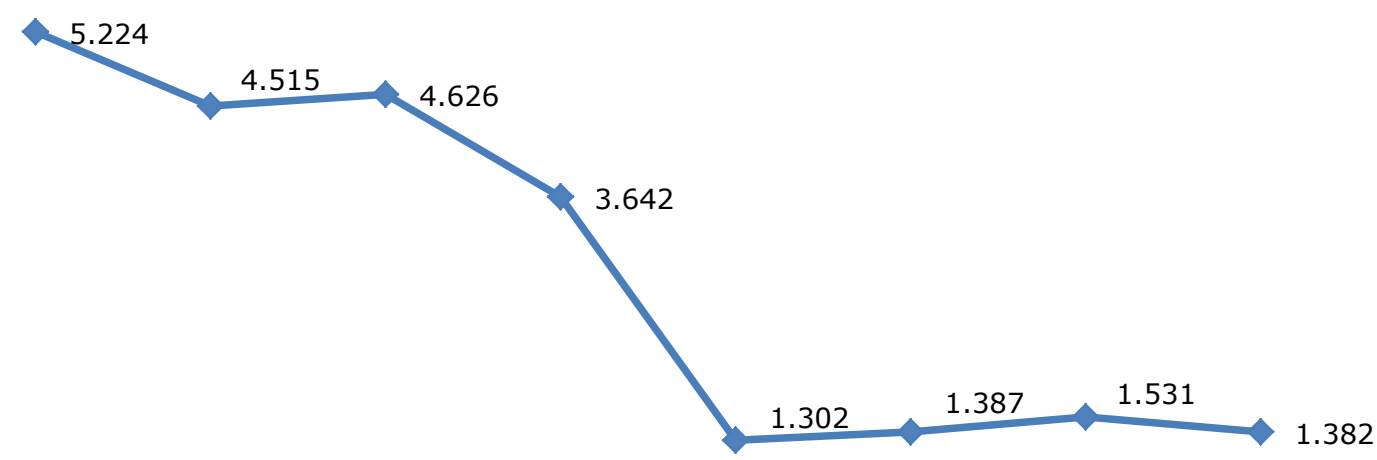

ago/09 set/09 out/09 nov/09 dez/09 jan/10 fev/10 mar/10

Fonte: Núcleo de Informação do Hospital, Londrina, PR, 2010.

Já no PSORT e PSC a redução no número de consultas foi menor. Esta situação explica-se pelo fato de que a maioria das consultas realizadas no PSORT, pelo médico ortopedista, é solicitada pelos plantonistas da instituição. Em relação ao PCS, neste hospital realizamse cirurgias eletivas e, em muitos casos, os pacientes que apresentam alguma complicação no período pósoperatório buscam atendimento na instituição tendo o PSC como porta de entrada.
No PSORT, foram realizadas 1.343 consultas médicas anteriormente a implantação do acolhimento, em média 335 consultas médicas/mês e 11 consultas médicas/dia. Já no período após o a avaliação de risco, foram realizadas 748 consultas médicas, com média de 187 consultas médicas/mês e seis consultas médicas/dia (Gráfico 3). Redução equivalente a $45 \%$ nas consultas médicas ao compararmos os períodos.

Gráfico 3: Consultas médicas realizadas no pronto-socorro ortopédico de um hospital de média complexidade, entre os meses de agosto/2009 a março/2010. Londrina, PR.

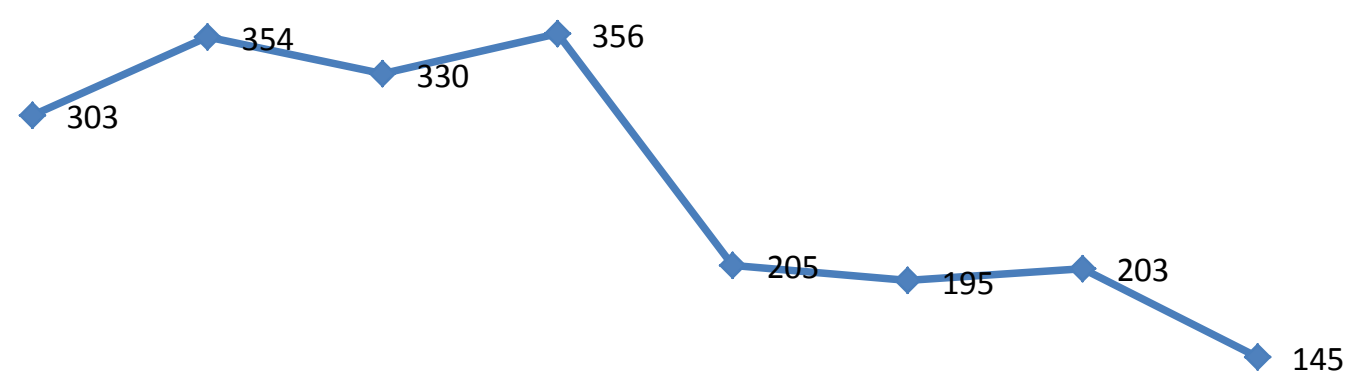

ago/09 set/09 out/09 nov/09 dez/09 jan/10 fev/10 mar/10

Fonte: Núcleo de Informação do Hospital, Londrina, PR, 2010.

No PSC, o número de consultas médicas realizadas no período avaliado, anterior ao AACR, foi 1.717 consultas, média de 429 consultas/mês e 14 consultas/dia. No período avaliado após o acolhimento, 
foram realizadas 1.403 consultas médicas, média 350

$19 \%$ das consultas médicas, comparando os dois consultas/mês e 11 consultas/dia. Houve redução de períodos (Gráfico 4).

Gráfico 4: Consultas médicas realizadas no pronto-socorro cirúrgico de um hospital de média complexidade, entre os meses de agosto/2009 a março/2010. Londrina, PR.

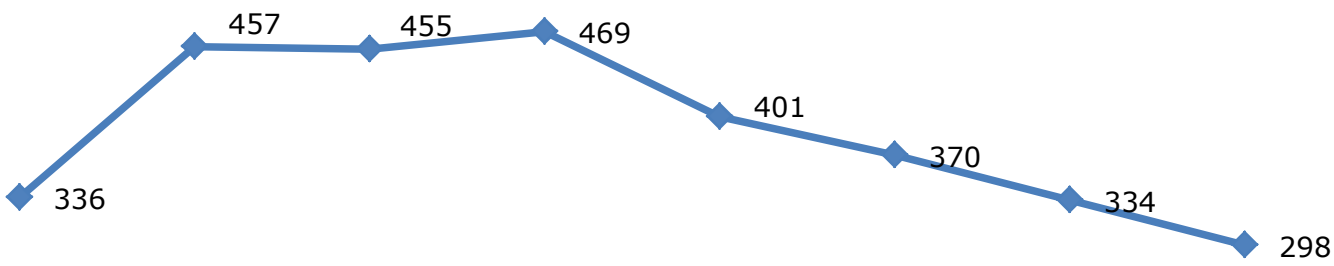

ago/09 set/09 out/09 nov/09 dez/09 jan/10 fev/10 mar/10

Fonte: Núcleo de Informação do Hospital, Londrina, PR, 2010.

Os dados do AACR revelaram que nos quatro primeiros meses após a implantação, 4.487 pessoas foram encaminhadas para outros serviços, ou seja, 36\% dos usuários passaram por consulta de enfermagem e foram encaminhados (Gráfico 5). Em média 1.121 usuários encaminhados/mês e 37 usuários encaminhados/dia.

Gráfico 5: Encaminhamentos a unidades básicas de saúde realizadas pelo enfermeiro da avaliação e classificação de risco do pronto-socorro de um hospital de média complexidade, entre os meses de agosto/2009 a março/2010. Londrina, PR.

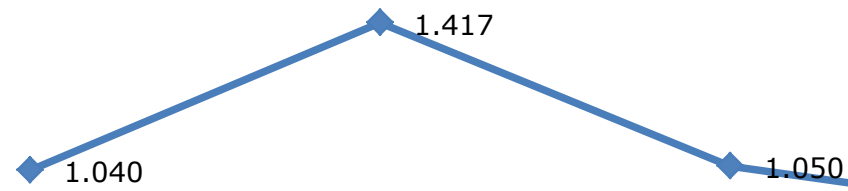

$\mathrm{fev} / 10$

$\operatorname{mar} / 10$

Fonte: Núcleo de Informação do Hospital, Londrina, PR, 2010.

A quantidade de usuários encaminhados para as UBS revela que grande parcela dos pacientes que buscam atendimento no pronto-socorro do hospital do estudo poderia ter seu problema resolvido na atenção primária ou ambulatorial. Neste serviço o maioria usuários busca a realização de exames e encaminhamentos para 
especialidades, pois encontram enormes filas nos agendamentos de exames e consultas médicas nas UBSs.

Entretanto, estes usuários não podem ser considerados como "inadequados" para instituição e encaminhados sem a garantia de que serão atendidos em outro serviço. A implantação do AACR deveria dar o destino correto a essa clientela, porém a população cada vez mais numerosa e com poucos recursos disponíveis na atenção básica encontra nos serviços de emergência o único lugar possível para atender as suas necessidades. Por isso, sem uma revisão e reestruturação da rede de atenção à saúde, os serviços emergência continuarão servindo como porta de entrada pelos usuários que não são atendidos na atenção primária e ambulatorial ${ }^{(14)}$.

Os serviços que compõe a rede de atenção à saúde deveriam discutir com a gestão local como integrar esse tipo de usuário às outras possíveis portas de entrada e prepará-las para atendê-los, já que as demandas são geradas por fatores culturais e por deficiências de recursos tecnológicos e sociais ${ }^{(14)}$.

É preciso substituir o modelo hospitalocêntrico por um modelo que estabeleça maior integração entre os serviços de saúde pela constatação que nenhum serviço, isoladamente, dispõe de recursos humanos, financeiros, competência e tecnologia para fazer frente a atenção integral à saúde da população. O caminho para superar o modelo atual de atenção nos serviços de emergência deverá ser de caráter sistêmico e ter como foco o usuário, com redefinição e integração das vocações assistenciais, reorganização de fluxos e repactuação do processo de trabalho ${ }^{(7)}$.

Além disso, é necessária a superação da percepção da população e de muitos profissionais de saúde que acreditam que a assistência complexa e eficaz é aquela oferecida nos hospitais com exames diagnósticos e procedimentos complexos, desqualificando o papel da atenção primária. Entre os profissionais de saúde esta percepção decorre, em grande medida, de uma formação profissional centrada no espaço hospitalar e no modelo da biomedicina. Isto tem dificultado a compreensão de que a atenção primária tem uma grande complexidade, demandando conhecimentos e práticas diferentes da atenção produzida em outros locais ${ }^{(16)}$

A queda significativa no número de consultas médicas e também na quantidade de usuários que buscaram atendimento nesta instituição levantaram as questões: Os pacientes encaminhados buscaram atendimentos nos serviços aos quais foram referenciados? Será que foram acolhidos?

O AACR isoladamente não garante uma melhoria na qualidade da assistência, ao contrário, se o usuário encaminhado para outro serviço não for acolhido por este, acaba sendo excluído da rede de atenção à saúde e, muitas vezes, tem seu problema de saúde agravado pela demora no diagnóstico e no tratamento. Por isto é são tão importantes as pactuações internas e externas para a viabilização do processo, com a construção de fluxos claros por grau de risco, e a tradução destes na rede de atenção(2).

\section{CONCLUSÃO}

A estruturação do grupo de trabalho constituído por equipe multiprofissional e a construção do PES proporcionaram a operacionalização da implantação do AACR. Além disso, a participação dos profissionais da instituição, usuários e coordenadores de outros serviços de saúde auxiliaram na implementação do PES elaborado pelo grupo de trabalho o que viabilizou a instituição no novo fluxograma e a retomada das discussões sobre o sistema se referencia e contra-referencia no município.

Observou-se uma redução significativa nos atendimentos médicos após a avaliação e classificação de risco e uma grande quantidade de encaminhamentos de usuários a outros serviços. Este fato tornou mais evidente a demanda reprimida de outros setores, composta, principalmente, por usuários não atendidos na atenção primária e os que aguardam procedimentos especializados.

Vale ressaltar que a implantação do AACR proporcionou maior agilidade no atendimento de pacientes com quadro clínico grave, como risco de morte, ao capacitar profissionais a identificar sinais e sintomas que indicam situações de urgência e emergência e que precisam ser priorizadas.

Os prontos-socorros dos hospitais têm por função atender urgências e emergências, por isto o gestor deve garantir que o usuário receba assistência adequada o mais rápido o possível, evitando-se o óbito e minimizando sequelas ao paciente.

Dessa forma, não é admissível que se mantenha os atendimentos por ordem de chegada, sem priorizar indivíduos em real situação de urgência/emergência, por conta da superlotação causada pela busca desordenada de usuários por problemas estruturais da rede de atenção à saúde. 
Levantou-se a necessidade de que outras pesquisas sejam realizadas para avaliar o impacto do AACR na rede de serviços de saúde e as facilidades e/ou dificuldades de acesso encontradas pelos usuários na busca pela assistência.

\section{REFERÊNCIAS}

1. Ministério da Saúde; Secretaria de Atenção à Saúde; Núcleo Técnico da Política Nacional de Humanização. HumanizaSUS: Documento base para gestores e trabalhadores do SUS. Brasília (Brasil): Ministério da Saúde; 2008.

2. Ministério da Saúde. Secretaria de Atenção à Saúde. Política Nacional de Humanização da Atenção e Gestão do SUS. Acolhimento e classificação de risco nos serviços de urgência. Brasília (Brasil): Ministério da Saúde; 2009.

3. Almeida PJS, Pires DEP. O trabalho em emergência: entre o prazer e o sofrimento. Rev. Eletr. Enf. [Internet]. 2007 [cited 2011 dez 29];9(3):617-29. Available from: http://www.fen.ufg.br/revista/v9/n3/v9n3a05.htm.

4. O’Dwyer G, Oliveira SP, Seta MH. Avaliação dos serviços hospitalares de emergência do programa QualiSUS. Cien Saude Colet. 2009;14(5):1881-90.

5. Castilho Sá M, Carreteiro TC, Fernandes MIA. Limites do cuidado: representações e processos inconscientes sobre a população na porta de entrada de um hospital de emergência. Cad Saude Publica. 2008;24(6):1334-43.

6. Ministério da Saúde; Secretaria-Executiva; Núcleo Técnico da Política Nacional de Humanização. HumanizaSUS: acolhimento com avaliação e classificação de risco: um paradigma éticoestético no fazer em saúde. Brasília (Brasil): Ministério da Saúde; 2004.

7. Bittencourt RJ, Hortale VA. A qualidade nos serviços de emergência de hospitais públicos e algumas considerações sobre a conjuntura recente no município do Rio de Janeiro. Cien Saude Colet. 2007; 12(4):929-34.

8. Medeiros AC, Pereira QLC, Siqueira HCH, CecagnoI D, Moraes CL. Gestão participativa na educação permanente em saúde: olhar das enfermeiras. Rev Bras Enferm. 2010; 63(1):38-42.

9. Melleiro MM, Tronchin DMR, Ciampone MHT. O planejamento estratégico situacional no ensino do gerenciamento em enfermagem. Acta paul. enferm. 2005;18(2):165-71.

10. Backes DS, Lunardi Filho WD, Lunardi VL. O processo de humanização do ambiente hospitalar centrado no trabalhador. Rev Esc Enferm USP. 2006;40(2):221-7.

11. Bittencourt RJ, Hortale VA. Intervenções para solucionar a superlotação nos serviços de emergência hospitalar: uma revisão sistemática. Cad Saude Publica. 2009;25(7): 1439-54.

12. Oliveira BRG, Collet N, Viera CS. A humanização na assistência à saúde. Rev Lat Am Enfermagem. 2006;14(2):27784.

13. Souza CC, Toledo AD, Tadeu LFR, Chianca TCM. Classificação de risco em pronto-socorro: concordância entre protocolo institucional brasileiro e Manchester. Rev Lat Am Enfermagem. $2011 ; 19(1): 26-33$.

14. Nascimento ERP, Hilsendeger BR, Neth C, Belaver GM, Bertoncello KCG. Classificação de risco na emergência: avaliação da equipe de enfermagem. Rev. enferm. UERJ. 2011;19(1):84-8. 15. O'Dwyer G, Matta IEA, Pepe VLE. Avaliação dos serviços hospitalares de emergência do estado do Rio de Janeiro. Cien Saude Colet. 2008;13(5):1637-48.

16. Ministério da Saúde. Secretaria de Atenção à Saúde. Política Nacional de Humanização da Atenção e Gestão do SUS. O HumanizaSUS na atenção básica. Brasília (Brasil): Ministério da Saúde; 2009.

Artigo recebido em 15.06.2010.

Aprovado para publicação em 17.11.2011.

Artigo publicado em 31.12.2011. 